\title{
Molecular Identification and Quantification of Tetracycline and Erythromycin Resistance Genes in Spanish and Italian Retail Cheeses
}

\author{
Ana Belén Flórez, ${ }^{1}$ Ángel Alegría, ${ }^{1,2}$ Franca Rossi, ${ }^{2}$ Susana Delgado, ${ }^{1}$ \\ Giovanna E. Felis, ${ }^{2}$ Sandra Torriani, ${ }^{2}$ and Baltasar Mayo ${ }^{1}$ \\ ${ }^{1}$ Departamento de Microbiología y Bioquímica, Instituto de Productos Lácteos de Asturias (IPLA-CSIC), Paseo Río Linares s/n, \\ Villaviciosa, 33300 Asturias, Spain \\ ${ }^{2}$ Dipartimento di Biotecnologie, Università Degli Studi di Verona, Strada Le Grazie, 15, 37134 Verona, Italy \\ Correspondence should be addressed to Baltasar Mayo; baltasar.mayo@ipla.csic.es
}

Received 4 July 2014; Revised 21 August 2014; Accepted 27 August 2014; Published 11 September 2014

Academic Editor: Gundlapally S. Reddy

Copyright @ 2014 Ana Belén Flórez et al. This is an open access article distributed under the Creative Commons Attribution License, which permits unrestricted use, distribution, and reproduction in any medium, provided the original work is properly cited.

\begin{abstract}
Large antibiotic resistance gene pools in the microbiota of foods may ultimately pose a risk for human health. This study reports the identification and quantification of tetracycline- and erythromycin-resistant populations, resistance genes, and gene diversity in traditional Spanish and Italian cheeses, via culturing, conventional PCR, real-time quantitative PCR (qPCR), and denaturing gradient gel electrophoresis (DGGE). The numbers of resistant bacteria varied widely among the antibiotics and the different cheese varieties; in some cheeses, all the bacterial populations seemed to be resistant. Up to eight antibiotic resistance genes were sought by gene-specific PCR, six with respect to tetracycline, that is, $\operatorname{tet}(\mathrm{K}), \operatorname{tet}(\mathrm{L}), \operatorname{tet}(\mathrm{M}), \operatorname{tet}(\mathrm{O}), \operatorname{tet}(\mathrm{S})$, and tet $(\mathrm{W})$, and two with respect to erythromycin, that is, $\operatorname{erm}(\mathrm{B})$ and $\operatorname{erm}(\mathrm{F})$. The most common resistance genes in the analysed cheeses were tet $(\mathrm{S})$, tet $(\mathrm{W})$, tet $(\mathrm{M})$, and $\operatorname{erm}(\mathrm{B})$. The copy numbers of these genes, as quantified by qPCR, ranged widely between cheeses (from 4.94 to $10.18 \mathrm{log}_{10} / \mathrm{g}$ ). DGGE analysis revealed distinct banding profiles and two polymorphic nucleotide positions for tet(W)-carrying cheeses, though the similarity of the sequences suggests this tet $(\mathrm{W})$ to have a monophyletic origin. Traditional cheeses would therefore appear to act as reservoirs for large numbers of many types of antibiotic resistance determinants.
\end{abstract}

\section{Introduction}

Antibiotic resistance (AR) is a natural phenomenon, the appearance of which predates the clinical use of antibiotics $[1,2]$. Unfortunately, the widespread use and misuse of antibiotics in clinical and nonclinical environments for more than seven decades have provided optimal conditions for the appearance, mobilization, and concentration of highly efficient resistance systems in bacteria [3]. The transfer of AR genes into human and animal pathogens could ultimately lead to a failure of antibiotic therapy [4]. Mobilization among bacterial species is facilitated by AR genes being commonly located on mobile genetic elements such as transposons and plasmids, which have high horizontal transfer capacity [5]. The presence of antibiotics in the environment not only provides a positive selection for resistant pathogens, but also exerts an evolutive pressure on components of the commensal microbiota [6]. Under these conditions, the commensal bacteria in food could become a reservoir for AR determinants that could then further be disseminated via the food chain [7-9].

Determining the prevalence of AR genes in a given environment, and their characterization, requires the isolation and identification of the resistant bacteria, followed by a molecular analysis of their AR determinants [10-12]. The identification and quantification of AR genes directly in environmental samples, that is, without culturing biases, would be useful $[13,14]$. Culture-independent analysis is also faster and more accurate than culture-based methods. Indeed, several $A R$ identification and AR gene quantification techniques 
that require no culturing have recently been developed, such as denaturing gradient gel electrophoresis (DGGE) $[15,16]$, real-time quantitative PCR (qPCR) $[13,14,17]$, construction and functional analysis of gene libraries [18-20], AR gene microarrays [21, 22], and analysis of metagenomic sequences $[23,24]$. Knowledge of the types and loads of AR genes in foods could ultimately be of help to estimate the risk of their transmission during cheese manufacturing and after consumption. To our knowledge, few attempts have been made to directly analyze AR gene numbers and diversity in food samples.

The aims of the present work were to identify the microbial populations resistant to tetracycline and erythromycin in commercial Spanish and Italian cheeses and to quantify their antibiotic resistance genes, using culturing and molecular techniques. These antibiotics were selected as a model due to the fact that resistance against tetracycline and erythromycin has been extensively documented among food-borne bacteria, including strains of lactic acid bacteria species.

\section{Material and Methods}

2.1. Cheese Sampling. Twenty commercial cheeses-10 Spanish (Cabrales, Zamorano, Majorero, Mahón, Torta del Casar, Manchego, Ibores, Garrotxa, De La Mesta, and Ibérico) and 10 Italian (Gorgonzola "dolce," Gorgonzola "picante," Caprino, Quartirolo Lombardo, Pecorino Sardo, Grana Padano, Montasio, Monte Veronese, Asiago, and Taleggio)were bought at retail stores. These cheese types vary in terms of the technology used to manufacture them (artisanal (without starters), industrial (using commercial starters), the type of milk used (cow, goat, ewe, or mixtures), the treatment to which that milk is subject (raw, pasteurised), and ripening time (from one to 24 months)).

2.2. Plate Counts of Cultivable Antibiotic-Resistant Bacteria. Cubes of ten grams of cheese from the centre were homogenised with $90 \mathrm{~mL}$ of a $2 \%(\mathrm{w} / \mathrm{v})$ sterilised sodium citrate solution prewarmed at $45^{\circ} \mathrm{C}$ for $1 \mathrm{~min}$ in a Colworth Stomacher 400 (Seward Ltd., London, UK). Cheese homogenates were tenfold diluted in Ringer's solution (Merck, Darmstadt, Germany) and the dilutions were plated in duplicate on selective and nonselective agarified media. Total aerobic mesophilic bacteria (including aerotolerant and facultative anaerobes) were enumerated on Plate Count Milk Agar (PCMA; Merck), Gram-positive bacteria such as lactic acid bacteria (LAB) were enumerated using de Man Rogosa and Sharpe agar (MRS; Merck), enterococci on Slanetz and Bartley agar (SB; Merck), and staphylococci on Baird-Parker agar (BP; Merck). Tetracycline and erythromycin resistant populations were counted using the same nonselective selective and media containing tetracycline $\left(15 \mu \mathrm{g} \mathrm{mL}^{-1}\right)$ and erythromycin $\left(8 \mu \mathrm{g} \mathrm{mL}^{-1}\right)$ (Sigma-Aldrich, St. Louis, MO, USA). Antibiotic concentrations were considered eight- to tenfolds higher than the usual resistance levels of susceptible populations [29]. This concentration, which is much lower than that provided by dedicated resistance mechanisms [5, 29], was considered to avoid unspecific growth of susceptible bacteria. Plates were then incubated at $30^{\circ} \mathrm{C}$ (PCMA and
MRS media) and $45^{\circ} \mathrm{C}$ (SB medium) for $72 \mathrm{~h}$ and at $37^{\circ} \mathrm{C}(\mathrm{BP}$ medium) for $48 \mathrm{~h}$.

2.3. Total DNA Extraction and Purification. Cheese samples (5 grams) were homogenized with $45 \mathrm{~mL}$ of a $2 \%$ sterile sodium citrate solution and incubated at $37^{\circ} \mathrm{C}$ for $3 \mathrm{~h}$ in the presence of $1 \mathrm{mg} \mathrm{mL}^{-1}$ pronase (Sigma-Aldrich) and $100 \mu \mathrm{L}$ $\beta$-mercaptoethanol (Merck). Total microbial cells were harvested by centrifugation at $5000 \times \mathrm{g}$ for $20 \mathrm{~min}$ and disrupted using $0.1 \mathrm{~mm}$ glass beads (Sigma-Aldrich) as reported elsewhere [30]. Genomic DNA was purified by phenol and phenol/chloroform extractions and precipitated with 2-propanol (all chemicals from Sigma-Aldrich). Finally, it was suspended in sterile water containing $5-15 \mathrm{mg} \mathrm{mL}^{-1}$ of RNase (Sigma-Aldrich).

Total DNA was also isolated from pure cultures of control strains carrying known AR genes: Staphylococcus epidermidis SE36 [tet $(\mathrm{K})]$, Enterococcus faecium ET51 [tet $(\mathrm{L})]$, Lactococcus lactis IPLA 31008 [tet(M)], Enterococcus faecalis Jtet $[$ tet $(\mathrm{O})]$, Enterococcus spp. ET15 [tet(S)], Bifidobacterium longum B93 [tet(W)], Lactobacillus johnsonii G41 [erm(B)], and Bacteroides fragilis $79 \mathrm{a}[\operatorname{erm}(\mathrm{F})]$. Genomic microbial DNA from these strains was purified from $1 \mathrm{~mL}$ of an overnight culture in brain heart infusion broth (BHI; Merck) using the Kit GenElute bacterial genomic DNA (SigmaAldrich). The recovered DNA was then stored at $-20^{\circ} \mathrm{C}$ until analysis.

2.4. PCR Detection of Tetracycline and Erythromycin Resistance Genes. The presence of tetracycline and erythromycin resistance genes was examined in DNA from the cheese samples and from control strains by standard PCR using both universal primers for genes encoding tetracycline resistance through ribosomal protection proteins (RPPs), such as tet $(\mathrm{M})$, tet $(\mathrm{O})$, tet $(\mathrm{S})$, and tet $(\mathrm{W})$, and gene-specific primer pairs for genes encoding tetracycline resistance $[$ tet $(\mathrm{K})$ and tet $(\mathrm{L})]$ and erythromycin resistance $[\operatorname{erm}(\mathrm{B})$ and $\operatorname{erm}(\mathrm{F})]$ (Table 1). The PCR conditions used for amplification were those reported in Table 1. Positive (DNA from appropriate control strains) and negative (no template DNA) controls were subjected to amplification under the same conditions.

\subsection{Real-Time Quantitative PCR}

2.5.1. Design of Primers. Updated tetracycline and erythromycin resistance genes in databases were compiled for designing of new primers for the qPCR analysis. Nucleotide sequences encoding tetracycline resistance in the form of RPPs and efflux pumps, and erythromycin resistance, were downloaded from the GenBank database (see Table 1 in Supplementary Material available online at http://dx.doi.org/ $10.1155 / 2014 / 746859)$ and aligned with one another using Mega 5 software. Conserved regions at appropriate distances were used for designing the primers employed in qPCR reactions (Table 1). This was achieved using Primer Express 3.0 software (Applied Biosystems, Carlsbad, CA, USA).

2.5.2. qPCR Conditions. $\mathrm{qPCR}$ analyses were performed to quantify tetracycline (tet) and erythromycin ( $\mathrm{erm})$ resistance 
TABLE 1: Sequence and properties of the PCR primers used in this work.

\begin{tabular}{|c|c|c|c|c|c|}
\hline Application/primer & Target gene & Sequence $\left(5^{\prime}-3^{\prime}\right)$ & Annealing $T^{\mathrm{a}}\left({ }^{\circ} \mathrm{C}\right)$ & Amplicon size (bp) & Reference \\
\hline \multicolumn{6}{|l|}{ Conventional PCR } \\
\hline Tet-F & \multirow{2}{*}{ tet ${ }^{\mathrm{a}}$} & GCTCA(T/C)GTTGA(T/C)GCAGGAA & \multirow{2}{*}{50} & \multirow{2}{*}{1292} & \multirow{2}{*}[25]{} \\
\hline Tet-R & & AGGATTTGGCGG(C/G)ACTTC(G/T)A & & & \\
\hline TetK-F & \multirow{2}{*}{ tet $(\mathrm{K})$} & TTATGGTGGTTGTAGCTAGAAA & \multirow{2}{*}{55} & \multirow{2}{*}{348} & \multirow{2}{*}[15]{} \\
\hline TetK-R & & AAAGGGTTAGAAACTCTTGAAA & & & \\
\hline TetL-F & \multirow{2}{*}{$\operatorname{tet}(\mathrm{L})$} & GTMGTTGCGCGCTATATTCC & \multirow{2}{*}{55} & \multirow{2}{*}{696} & \multirow[t]{2}{*}[15]{} \\
\hline TetL-R & & GTGAAMGRWAGCCCACCTAA & & & \\
\hline DI-F & \multirow{2}{*}{$\operatorname{tet}(\mathrm{M})$} & GAYACICCIGGICAYRTIGAYTT & \multirow{2}{*}{55} & \multirow{2}{*}{1513} & \multirow{2}{*}{ [26] } \\
\hline TetM-R & & CACCGAGCAGGGATTTCTCCAC & & & \\
\hline TetO-F & \multirow{2}{*}{$\operatorname{tet}(\mathrm{O})$} & AATGAAGATTCCGACAATTT & \multirow{2}{*}{55} & \multirow{2}{*}{781} & \multirow{2}{*}{ [26] } \\
\hline TetO-R & & CTCATGCGTTGTAGTATTCCA & & & \\
\hline TetS-F & \multirow{2}{*}{ tet $(\mathrm{S})$} & ATCAAGATATTAAGGAC & \multirow{2}{*}{55} & \multirow{2}{*}{573} & [26] \\
\hline TetS-R & & TTCTCTATGTGGTAATC & & & \\
\hline TetW-F & $\operatorname{tet}(\mathrm{W})$ & AAGCGGCAGTCACTTCCTTCC & 50 & 1150 & [11] \\
\hline Tet-R & & AGGATTTGGCGG(C/G)ACTTC(G/T)A & 30 & 110 & {$[11]$} \\
\hline ErmB-F & $\operatorname{erm}(\mathrm{B})$ & GAAAAGGTACTCAACCAAATA & 50 & 639 & {$[27]$} \\
\hline ErmB-R & cim(D) & AGTAACGGTACTTAAATTGTTTAC & & (2) & \\
\hline ErmF-F & $\operatorname{erm}(\mathrm{F})$ & CGGGTCAGCACTTTACTATTG & 50 & 466 & [27] \\
\hline ErmF-R & (1) & GGACCTACCTCATAGACAAG & 50 & 700 & \\
\hline Real-time qPCR & & & & & \\
\hline TBA-F & $16 \mathrm{~S} r \mathrm{DNA}$ & CGGCAACGAGCGCAACCC & 60 & 130 & {$[28]$} \\
\hline TBA-R & $1001 D N A$ & CCATTGTAGCACGTGTGTAGCC & 60 & 150 & {$[20]$} \\
\hline TetK-qPCR-F & $\operatorname{tet}(\mathrm{K})$ & TGCTGCATTCCCTTCACTGA & 60 & 69 & This study \\
\hline TetK-qPCR-R & & GCTTTGCCTTGTTTTTTTCTTGTAA & & & \\
\hline TetL-qPCR-F & $\operatorname{tet}(\mathrm{L})$ & GGGTAAAGCATTTGGTCTTATTGG & 60 & 63 & This study \\
\hline tetL-qPCR-R & & ATCGCTGGACCGACTCCTT & & & \\
\hline TetM-qPCR-F & $\operatorname{tet}(\mathrm{M})$ & CAGAATTAGGAAGCGTGGACAA & 60 & 67 & This study \\
\hline TetM-qPCR-R & & CСTCTCTGACGTTCTAAAAGCGTAT & & & \\
\hline TetO-qPCR-F & $\operatorname{tet}(\mathrm{O})$ & AATGTCAGAACTGGAACAGGAAGAA & 60 & 59 & This study \\
\hline TetO-qPCR-R & & CGTGATAAACGGGAAATAACGTT & & & \\
\hline TetS-qPCR-F & tet $(\mathrm{S})$ & CGAGGTCATTCTCATTGGTGAA & 60 & 84 & This study \\
\hline TetS-qPCR-R & & CAGACACTGCGTCCATTTGTAAA & & $0 T$ & 1110 stuity \\
\hline TetW-qPCR-F & $\operatorname{tet}(\mathrm{W})$ & ACGGCAGCGCAAAGAGAA & 60 & 60 & This study \\
\hline TetW-qPCR-R & & CGGGTCAGTATCCGCAAGTT & & & \\
\hline ErmB-qPCR-F & $\operatorname{erm}(\mathrm{B})$ & GGATTCTACAAGCGTACCTTGGA & 60 & 69 & This studd \\
\hline ErmB-qPCR-R & $\operatorname{erm}(\mathrm{D})$ & AATCGAGACTTGAGTGTGCAAGAG & 00 & 09 & Ims stuay \\
\hline ErmF-qPCR-F & $\operatorname{erm}(\mathrm{F})$ & TGATGCCCGAAATGTTCAAGT & 60 & 63 & This study \\
\hline ErmF-qPCR-R & & AAAGGAAATTTCGGAACTGCAA & & & \\
\hline DGGE assays & & & & & \\
\hline TetM-F & $\operatorname{tet}(\mathrm{M})$ & ACAGAAAGCTTATTATATAAC & 55 & 171 & {$[15]$} \\
\hline $\mathrm{GC}^{\mathrm{b}}-\mathrm{TetM}-\mathrm{R}$ & & TGGCGTGTCTATGATGTTCAC & & & \\
\hline TetW-F & $\operatorname{tet}(\mathrm{W})$ & GAGAGCCTGCTATATGCCAGC & 64 & 168 & [15] \\
\hline GC-TetW-R & $\operatorname{ces}(\mathrm{vi})$ & GGGCGTATCCACAATGTTAAC & 04 & 108 & \\
\hline
\end{tabular}

${ }^{\mathrm{a}}$ tet, genes encoding ribosome protection proteins.

${ }^{\mathrm{b}}$ Sequence of the GC clamp: CGCCCGGGGCGCGCCCCGGGCGGGGGGGGGGCACGGGGGG.

genes in the total microbial DNA from the cheese samples. Amplifications were performed in an ABI Prism Fast 7500 sequence detection system (Applied Biosystems). Each $20 \mu \mathrm{L}$ qPCR reaction included $4 \mu \mathrm{L}$ of extracted DNA (approximately $200 \mathrm{ng}$ ), $10 \mu \mathrm{L}$ of SYBR Green PCR Master Mix (containing ROX as a passive reference), and $900 \mathrm{nM}$ of each primer. The following cycling conditions were used: $2 \mathrm{~min}$ at $50^{\circ} \mathrm{C}$, an initial denaturation step of $10 \mathrm{~min}$ at $95^{\circ} \mathrm{C}$, followed by 40 cycles of $15 \mathrm{~s}$ at $95^{\circ} \mathrm{C}$, and $60 \mathrm{~s}$ at $60^{\circ} \mathrm{C}$. Baseline and threshold calculations were performed using ABI Prism 
Fast 7500 software. Melting temperature analyses and size estimations of the PCR products were performed on agarose gels to check for nonspecific amplification.

2.5.3. Construction of $q P C R$ Standards. Conventional PCR reactions, as described above, were used to generate genespecific amplicons used as template DNA standards for qPCR. Amplicons were purified using a gel/PCR extraction kit (ATP Biotech, Banciao City, Taiwan) and quantified fluorometrically using a Gen5 Microplate dsDNA Quantitation Kit (Biotek Instruments, Winooski, VT, USA). The number of DNA molecules was calculated based on the size and mass of the amplicons. Tenfold serial dilutions of the amplicons were prepared and used following the procedure by Yu et al. [17]. To ensure accuracy in copy number quantification, a complementary control standard curve was obtained using DNA from known numbers of B. longum B93 cells (enumerated in MRS agar plates containing $0.25 \%$ cysteine following incubation in anaerobiosis at $37^{\circ} \mathrm{C}$ for $72 \mathrm{~h}$ ); this strain carries a single copy of tet(W) [31]. Dilutions of total DNA from the cells were subjected to qPCR analysis as above.

2.5.4. qPCR Expression Data. Absolute gene copy number was expressed as the number of copies of resistant genes per $\mathrm{g}^{-1}$, whereas relative copy number was expressed as the number of resistant genes per million copies of total bacteria ribosomal genes, as determined with the universal prokaryotic primers TBA-F and TBA-R (Table 1). Absolute abundance was calculated based on the results obtained for the corresponding standard of each resistant gene. Relative abundance was calculated using $E^{\Delta \mathrm{Ct}}$, where $E$ is the efficiency of the primer according to the slope of standard curve ( $E=10^{-1 / \text { slope }}$ ) and $\Delta \mathrm{Ct}$ is the $\mathrm{Ct}$ value of the gene target (tetracycline and erythromycin resistance genes) normalized against the Ct value of the total bacterial numbers in the samples. For the relative quantification, the copy number of the $16 S$ rRNA genes per cell was averaged to five. The efficiency $E$ of each pair of primers was as follows: 1.94 for tet $(\mathrm{K}), 1.97$ for tet $(\mathrm{L}), 1.97$ for tet $(\mathrm{M}), 2,03$ for $\operatorname{tet}(\mathrm{O}), 2,02$ for tet $(\mathrm{S})$ and 2,03 for tet(W), and 1.94 for erm(B) and 1.99 for $\operatorname{erm}(\mathrm{F})$.

2.6. Denaturing Gradient Gel Electrophoresis Analysis. DNA from cheeses and strains harbouring known tet genes ( $\mathrm{Lac}$ tococcus lactis IPLA 31008 [tet(M)], Enterococcus faecalis Jtet $[$ tet $(\mathrm{O})]$, Enterococcus spp. ET15 [tet(S)], and Bifidobacterium longum B93 $[$ tet $(\mathrm{W})]$ ) was amplified by PCR using DGGE primers (Table 1), employing the PCR conditions described elsewhere [15]. DGGE analysis of the amplified tet genes was performed as previously reported [16] with slight modifications. Briefly, DGGE was performed in a DCode apparatus (Bio-Rad, Richmond, CA, USA) at $60^{\circ} \mathrm{C}$ on $8 \%$ polyacrylamide gels with a formamide-urea denaturing gradient of $15-50 \%$. Electrophoresis was conducted at $150 \mathrm{~V}$ for $2 \mathrm{~h}$ and $200 \mathrm{~V}$ for $1 \mathrm{~h}$. After electrophoresis, gels were stained in an ethidium bromide solution $\left(0.5 \mu \mathrm{g} \mathrm{mL}^{-1}\right)$, and the DNA bands were visualized and captured using a Gbox system and GeneSys software (Syngene, Cambridge, UK). After isolation and reamplification with the same primers without the GC clamp and identical PCR conditions, bands from the acrylamide gels were identified by sequencing and sequence comparison. Online similarity searches were performed by using the BLAST tool in the GenBank database (http://www.ncbi.nlm.nih.gov/BLAST/).

\section{Results}

3.1. Prevalence of $A R$ Populations in Spanish and Italian Cheeses. The Spanish and Italian cheese samples were subjected to conventional microbiological analysis on selective and differential media supplemented or not with tetracycline and erythromycin. Populations of tetracycline-resistant $\left(\operatorname{Tet}^{r}\right)$ and/or erythromycin-resistant bacteria $\left(\mathrm{Erm}^{\mathrm{r}}\right)$ were detected on most counting media for both Spanish and Italian cheeses made from raw or pasteurised milk (Tables 2 and 3, resp.). The AR bacterial counts varied widely depending on the microbial group and type of cheese. In general, Italian cheeses (Table 3) returned lower $\operatorname{Tet}^{\mathrm{r}}$ and Erm ${ }^{\mathrm{r}}$ counts than Spanish cheeses (Table 2). Table 2 shows the total number of mesophilic, aerobic, $\operatorname{Tet}^{\mathrm{r}}$, and $\mathrm{Erm}^{\mathrm{r}}$ bacteria (enumerated in PCMA) to reach a maximum of $6.79 \log _{10} \mathrm{cfu} \mathrm{g}^{-1}$ and $7.15 \log _{10} \mathrm{cfu} \mathrm{g}^{-1}$, respectively, in samples of Cabrales. Microbial counts in this cheese type showed the antibiotic-resistant populations to generally be two to three $\log _{10}$ units lower than in antibiotic-free media; such results were only returned by this cheese.

3.2. Prevalence of $A R$ Genes in Spanish and Italian Cheeses. Table 4 shows the resistance genes identified by PCR in total DNA from the different cheeses using specific primers for six tetracycline and two erythromycin resistance genes. Two or more AR genes were detected in all cheeses with the exception of Mahón and Gorgonzola "dolce" cheeses, both of which returned a single AR gene (tet $(\mathrm{W})$ and tet $(\mathrm{K})$, resp.). Indeed, the Spanish De La Mesta cheese harboured all eight AR genes examined in this work, while the Italian Asiago cheese harboured seven (all but tet $(\mathrm{L})$ ). The most common gene encoding tetracycline resistance was tet $(\mathrm{S})$, which was identified in nine Spanish and six Italian cheeses, followed by tet (W) in seven Spanish and four Italian cheeses. In contrast, tet $(\mathrm{L})$ and $\operatorname{tet}(\mathrm{O})$ were the least widespread of the tetracycline resistance determinants; these were detected in three Spanish cheeses and in two Spanish and two Italian cheeses, respectively. The $\operatorname{erm}(\mathrm{B})$ gene was detected in all but three cheese types, while the erm $(F)$ gene was identified in seven of the 10 Italian cheeses, but only in one Spanish cheese (De La Mesta).

3.3. Quantification of Tetracycline and Erythromycin Resistance Genes by qPCR. Samples positive for AR genes in gene-specific PCR were subjected to qPCR to quantify those identified. A standard curve encompassing $10^{3}$ through $10^{10}$ gene copies per reaction was produced using total DNA from B. longum B93 as a control $\left(r^{2} \geq 0.996\right)$. The qPCR detection limit determined from both the control DNA of B. longum and the serial dilutions of gene-specific amplicons was estimated to be about $10^{4}$ copies per gram of cheese. Independent reactions were performed in triplicate; high 
TABLE 2: Microbial counts $\left(\log _{10} \mathrm{cfu} \mathrm{g}^{-1}\right)$ of the total and antibiotic resistant bacterial populations found in samples of ten Spanish cheeses.

\begin{tabular}{|c|c|c|c|c|c|c|c|c|c|c|}
\hline \multirow{2}{*}{$\begin{array}{l}\text { Culture } \\
\text { medium }^{a}\end{array}$} & \multicolumn{10}{|c|}{ Cheese } \\
\hline & Cabrales* & Zamorano* & Majorero* & Mahón & Torta del Casar* & Manchego & Ibores* & Garrotxa & De La Mesta* & Ibérico \\
\hline PCMA & 7.74 & 6.64 & 9.23 & 6.49 & 8.18 & 8.21 & 8.26 & 7.58 & 6.01 & 8.62 \\
\hline $\mathrm{PCMA}+\mathrm{Tc}$ & 6.76 & 2.74 & 4.26 & $<2.0^{\mathrm{b}}$ & 5.98 & 3.21 & 5.76 & 3.68 & 5.46 & 4.70 \\
\hline PCMA + Erm & 7.15 & 3.92 & 4.36 & 3.84 & 5.82 & 3.16 & 5.65 & 4.72 & 3.91 & 3.62 \\
\hline MRS & 7.20 & 6.53 & 4.53 & 6.36 & 7.93 & 6.21 & 8.12 & 7.40 & 5.08 & 7.92 \\
\hline $\mathrm{MRS}+\mathrm{Tc}$ & 6.79 & 3.64 & 4.11 & $<2.0$ & 4.96 & $<2.0$ & 4.77 & 3.12 & $<2.0$ & $<2.0$ \\
\hline MRS + Erm & 6.90 & 4.18 & 4.03 & $<2.0$ & 4.89 & $<2.0$ & 5.67 & 3.22 & $<2.0$ & $<2.0$ \\
\hline $\mathrm{SB}$ & 5.83 & 4.07 & 2.95 & $<2.0$ & 5.67 & $<2.0$ & 5.85 & 3.13 & 4.82 & 5.04 \\
\hline $\mathrm{SB}+\mathrm{Tc}$ & 5.08 & 2.30 & $<2.0$ & $<2.0$ & 4.82 & $<2.0$ & 3.20 & $<2.0$ & 2.81 & 4.24 \\
\hline $\mathrm{SB}+$ Erm & 5.18 & 3.04 & $<2.0$ & $<2.0$ & 4.95 & $<2.0$ & $<2.0$ & $<2.0$ & 3.61 & $<2.0$ \\
\hline $\mathrm{BP}$ & 5.34 & 3.53 & 3.91 & 3.74 & 5.30 & $<2.0$ & 4.27 & 4.26 & 4.18 & 4.72 \\
\hline $\mathrm{BP}+\mathrm{Tc}$ & 4.71 & 2.78 & $<2.0$ & $<2.0$ & 4.84 & $<2.0$ & 2.98 & 3.20 & $<2.0$ & $<2.0$ \\
\hline $\mathrm{BP}+\mathrm{Erm}$ & 5.00 & 2.98 & 3.56 & $<2.0$ & 4.95 & $<2.0$ & $<2.0$ & 4.47 & $<2.0$ & $<2.0$ \\
\hline
\end{tabular}

${ }^{a}$ Culture media used for counting of different bacteria groups: PCMA for total mesophilic bacteria, MRS for lactic acid bacteria, SB for enterococci, and BP for staphylococci and micrococci. Antibiotics utilized to supplement the culture media: Tc, tetracycline and Erm, erythromycin.

${ }^{\mathrm{b}}$ Counts below the detection limit $\left(2.0 \log _{10} \mathrm{cfu} \mathrm{g}^{-1}\right)$.

${ }^{*}$ Cheeses made from raw milk.

TABLE 3: Microbial counts $\left(\log _{10} \mathrm{cfu} \mathrm{g}^{-1}\right)$ of the total and antibiotic resistant bacterial populations found in samples of ten Italian cheeses.

\begin{tabular}{|c|c|c|c|c|c|c|c|c|c|c|}
\hline \multirow{2}{*}{$\begin{array}{l}\text { Culture } \\
\text { medium }^{a}\end{array}$} & \multicolumn{10}{|c|}{ Cheese } \\
\hline & $\begin{array}{l}\text { Gorgonzola } \\
\text { "dolce" }\end{array}$ & $\begin{array}{l}\text { Gorgonzola } \\
\text { "piccante" }\end{array}$ & Caprino* & $\begin{array}{l}\text { Quartirolo } \\
\text { Lombardo }\end{array}$ & $\begin{array}{c}\text { Pecorino } \\
\text { Sardo* }^{*}\end{array}$ & $\begin{array}{c}\text { Grana } \\
\text { Padano* }\end{array}$ & Montasio* & $\begin{array}{c}\text { Monte } \\
\text { Veronese }\end{array}$ & Asiago* & Taleggio* \\
\hline PCMA & 7.78 & 8.70 & 8.23 & 6.00 & 6.00 & 8.04 & 7.38 & 8.63 & 7.36 & 8.34 \\
\hline $\mathrm{PCMA}+\mathrm{Tc}$ & 5.04 & 4.48 & 3.00 & 2.05 & $<2.0^{\mathrm{b}}$ & 3.60 & $<2.0$ & 3.00 & 4.60 & $<2.0$ \\
\hline PCMA + Erm & 6.00 & $<2.0$ & $<2.0$ & 2.85 & 5.30 & $<2.0$ & $<2.0$ & 5.45 & 2.48 & 2.70 \\
\hline MRS & 6.40 & 7.60 & 8.30 & 6.38 & 5.30 & 7.00 & 7.63 & 8.15 & 5.00 & 6.70 \\
\hline $\mathrm{MRS}+\mathrm{Tc}$ & 4.08 & $<2.0$ & $<2.0$ & $<2.0$ & $<2.0$ & 3.60 & 2.00 & $<2.0$ & 3.67 & $<2.0$ \\
\hline MRS + Erm & 3.08 & 3.01 & $<2.0$ & $<2.0$ & $<2.0$ & $<2.0$ & $<2.0$ & $<2.0$ & 3.73 & $<2.0$ \\
\hline SB & 5.46 & 5.21 & $<2.0$ & 6.00 & 4.88 & 3.60 & 4.41 & 3.20 & 5.30 & 2.00 \\
\hline $\mathrm{SB}+\mathrm{Tc}$ & $<2.0$ & $<2.0$ & $<2.0$ & 2.30 & $<2.0$ & 3.78 & 2.00 & 2.78 & 2.00 & $<2.0$ \\
\hline $\mathrm{SB}+$ Erm & 2.48 & $<2.0$ & $<2.0$ & $<2.0$ & 2.00 & $<2.0$ & 2.00 & $<2.0$ & $<2.0$ & $<2.0$ \\
\hline $\mathrm{BPA}$ & 5.95 & 5.44 & $<2.0$ & 3.90 & $<2.0$ & 3.97 & 2.60 & 3.78 & $<2.0$ & 2.85 \\
\hline $\mathrm{BP}+\mathrm{Tc}$ & 3.78 & 2.48 & $<2.0$ & 3.23 & $<2.0$ & 3.60 & 2.00 & $<2.0$ & $<2.0$ & $<2.0$ \\
\hline $\mathrm{BP}+\mathrm{Erm}$ & 3.00 & $<2.0$ & $<2.0$ & 2.48 & $<2.0$ & $<2.0$ & $<2.0$ & 3.00 & $<2.0$ & $<2.0$ \\
\hline
\end{tabular}

${ }^{a}$ Culture media used for counting of different bacteria groups: PCMA for total mesophilic bacteria, MRS for lactic acid bacteria, SB for enterococci, and BP for staphylococci and micrococci. Antibiotics utilized to supplement the culture media: Tc, tetracycline and Erm, erythromycin.

${ }^{\mathrm{b}}$ Counts below the detection limit $\left(2.0 \log _{10} \mathrm{cfu} \mathrm{g}^{-1}\right)$.

${ }^{*}$ Cheeses made from raw milk.

reproducibility was always obtained (average standard deviation $\leq 0.3$ ). The amplification of AR genes with conventional gene-specific PCR and qPCR primers returned similar results for the Spanish cheeses (Table 4; Figures 1 and 2). Indeed, only two samples showed qPCR amplification failures for genes previously detected by gene-specific PCR among the Spanish cheeses (one tet $(\mathrm{K})$ and one erm (B) gene). In contrast, among the Italian cheeses, eight samples showing AR genes in genespecific PCR analysis (three for erm (F), two for each erm(B) and tet $(\mathrm{W})$, plus one for tet $(\mathrm{S}))$ failed to show the same with qPCR-specific primers.
The copy number of the different tetracycline (Figure 1) and erythromycin (Figure 2) resistance genes varied widely among the cheese types. Absolute copy numbers of AR genes $\left(\log _{10} / \mathrm{g}\right.$ of cheese) ranged from $4.94(\mathrm{erm}(\mathrm{F})$ in Asiago cheese) to 10.18 (tet $(\mathrm{S})$ in Torta del Casar cheese). On average, the copy number of the different AR genes, quantified by qPCR analysis, was $6.6 \pm 1.2$ copies per gram of cheese. Figures 1 and 2 show the number of each resistance gene as a percentage (\%o) of the total bacterial loads in the cheeses as determined by $16 \mathrm{~S}$ rDNA analysis. In this way, intersample variations in gene copy number between cheeses are clearly 
TABLE 4: Tetracycline and erythromycin resistance genes detected by conventional PCR in Spanish and Italian cheeses.

\begin{tabular}{|c|c|c|c|c|c|c|c|c|}
\hline \multirow{3}{*}{ Cheese } & \multicolumn{8}{|c|}{ Gene } \\
\hline & \multicolumn{6}{|c|}{ Tetracycline resistance gene } & \multicolumn{2}{|c|}{ Erythromycin resistance gene } \\
\hline & $\operatorname{tet}(\mathrm{K})$ & $\operatorname{tet}(\mathrm{L})$ & $\operatorname{tet}(\mathrm{M})$ & $\operatorname{tet}(\mathrm{O})$ & tet $(\mathrm{S})$ & $\operatorname{tet}(\mathrm{W})$ & erm(B) & $\operatorname{erm}(\mathrm{F})$ \\
\hline \multicolumn{9}{|l|}{ Spanish cheeses } \\
\hline Cabrales* & + & + & + & - & + & + & + & - \\
\hline Zamorano* & + & + & + & - & + & + & + & - \\
\hline Majorero* & - & - & - & - & + & - & + & - \\
\hline Mahón & - & - & - & - & - & + & - & - \\
\hline Torta del Casar* & - & - & + & + & + & + & + & - \\
\hline Manchego & - & - & - & - & + & - & + & - \\
\hline Ibores $^{*}$ & - & - & + & - & + & + & + & - \\
\hline Garrotxa & - & - & - & - & + & + & + & - \\
\hline De La Mesta ${ }^{*}$ & + & + & + & + & + & + & + & + \\
\hline Ibérico & - & - & + & - & + & - & + & - \\
\hline \multicolumn{9}{|l|}{ Italian cheeses } \\
\hline Gorgonzola "dolce" & - & - & - & - & - & - & + & - \\
\hline Gorgonzola "piccante" & + & - & - & - & - & - & + & + \\
\hline Caprino* & - & - & - & + & + & + & + & + \\
\hline Quartirolo Lombardo & - & - & - & - & + & + & - & - \\
\hline Pecorino Sardo* & + & - & + & - & + & + & - & - \\
\hline Grana Padano* & - & - & - & - & - & - & + & + \\
\hline Montasio* & - & - & - & - & - & - & + & + \\
\hline Monte Veronese* & - & - & - & - & + & - & + & + \\
\hline Asiago ${ }^{*}$ & + & - & + & + & + & + & + & + \\
\hline Taleggio* & - & - & - & - & + & - & + & + \\
\hline
\end{tabular}

${ }^{*}$ Cheeses made from raw milk.

depicted. In general agreement with the microbial counting results, fewer AR gene copies were found in the Italian than in the Spanish cheeses.

3.4. Denaturing Gradient Gel Electrophoresis (DGGE) Analysis. DGGE analyses were undertaken to assess the intragenic sequence variability of two of the most prevalent AR genes, tet $(\mathrm{W})$ and tet $(\mathrm{M})$. Besides differences in gene copy number, sequence variability can be a cause of amplification failure in gene-specific PCR and qPCR. Figure 3 shows the DGGE profiles obtained for tet(W) using total DNA from Spanish cheese samples as a template. The DGGE primers returned amplifications in six of the seven cheese samples deemed positive by gene-specific PCR (all but Mahón cheese). A prominent band was present in all samples analysed, the mobility of which was comparable to that produced when using as a template DNA from the B. longum control strain carrying a chromosomal tet $(\mathrm{W})$ gene. Ten other bands of minor intensity were also observed across the cheeses (up to six bands in one sample; Figure 3, line 1), of which eight could be reamplified and sequenced. Sequence comparison showed these bands to be of identical nucleotide sequence to tet $(\mathrm{W})$, except at two polymorphic positions. These polymorphic bands migrated differently on the gel. However, surprisingly, some bands from different positions showed identical sequences.

DGGE analysis was also performed with specific primers for tet(M) using DNA from cheeses in which this gene had been identified. In this case, no differences were seen among the DGGE profiles. All the profiles obtained were composed of one intense and two weak bands, but all these bands were also produced when DNA from a control strain carrying a single-copy tet $(\mathrm{M})$ gene was used as a template (data not shown).

\section{Discussion}

It is acknowledged that the misuse and overuse of antibiotics in agriculture, aquaculture, animal husbandry, and the clinic have caused an increase in AR bacteria, the consequence of the selection pressure exerted in these environments $[3,32]$. $\mathrm{AR}$ in dairy products has traditionally been examined by conventional methods of microbial analysis. Indeed, in the present work, the tetracycline and erythromycin resistant populations of 20 Spanish and Italian traditional cheeses were first identified by culturing. High numbers of $\operatorname{Tet}^{\mathrm{r}}$ and Erm $^{\mathrm{r}}$ bacteria were found in most samples, suggesting cheese to be an important vehicle of AR genes within the food 


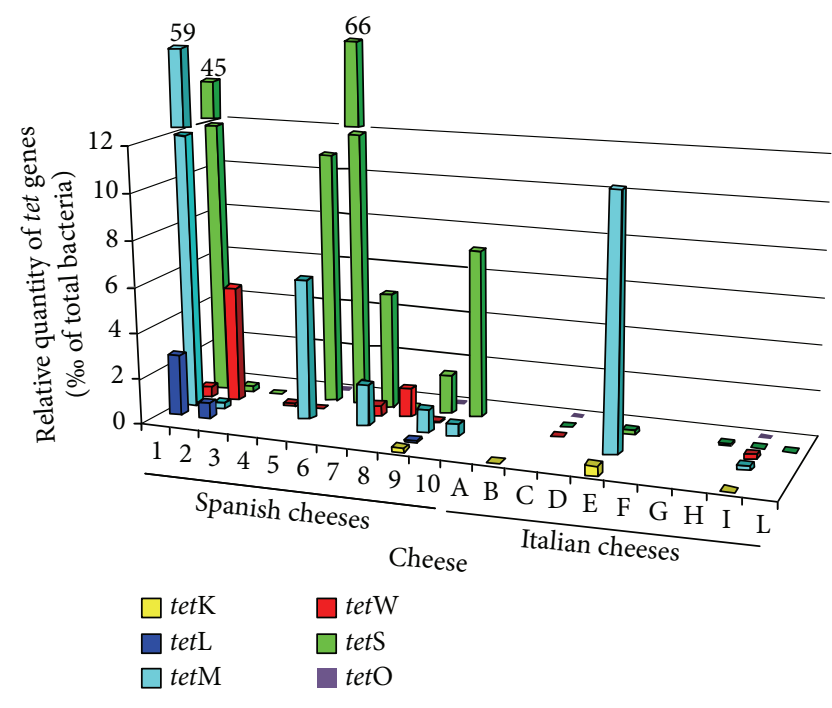

FIGURE 1: Bars diagram depicting the percentage copy number (\%o) of different tetracycline resistance genes quantified by qPCR as a function of the total bacteria in Spanish (1 to 10) and Italian (A to L) cheeses. The order of both Spanish and Italian cheese types is the same as in the tables.

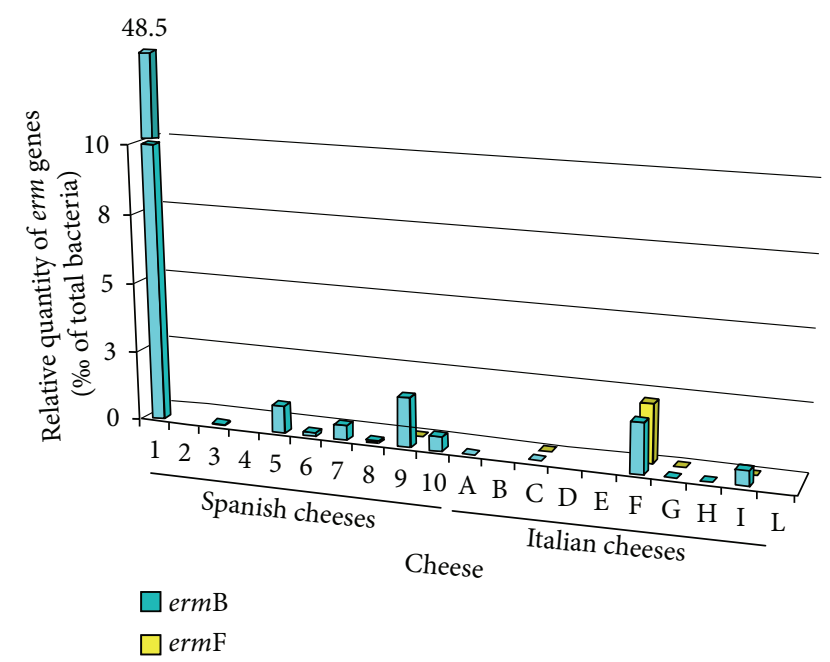

FIGURE 2: Bars diagram depicting the percentage copy number (\%o) of erm(B) and erm(F) erythromycin resistance genes quantified by qPCR as a function of the total bacteria in Spanish (1 to 10) and Italian (A to L) cheeses. The order of both Spanish and Italian cheese types is the same as in the tables.

chain. Similar ratios between counts of AR and antibiotic susceptible bacteria have previously been reported in other cheese types, such as Mozzarella [33] and Cheddar [8].

No significant differences were seen in AR counts between cheeses made from raw or pasteurised milk or between cheeses made from milk of different species. These results agree with previous reports that indicate reductions in the presence of AR bacteria only in sterile foods [4]. Pasteurisation may reduce the numbers and types of environmental bacteria, but traditional cheesemaking is open to contamination, in which organisms from many sources can

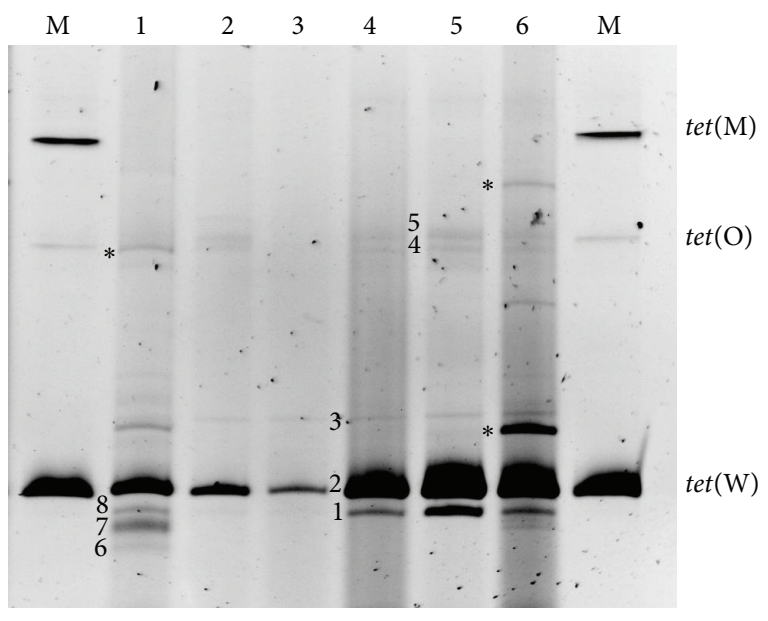

FIGURE 3: PCR-DGGE analysis of total DNA from Spanish cheeses using specific DGGE-primers for the tetracycline resistance tet $(\mathrm{W})$ gene. Lanes from 1 to 6, Cabrales, Zamorano, Torta del Casar, Ibores, Garrotxa, and De La Mesta. Lanes M, DGGE markers consisting of amplicons obtained with specific DGGE primers for tet $(\mathrm{M})$, tet $(\mathrm{O})$, and tet $(\mathrm{W})$, and using as template total DNA from bacterial strains harboring the respective tetracycline resistance gene. The asterisks denote bands that did no reamplification and, consequently, could not be identified.

gain access to and develop in the cheese. The Italian cheeses contained smaller AR populations than the Spanish ones. The curd-cooking step in some Italian cheeses may reduce the number of nonstarter microorganisms (e.g., enterococci and staphylococci), which have been shown to contain a plethora of resistance types [34-36]. Further, AR counts seemed to be inversely correlated with ripening time (data not shown), which is usually longer for Italian cheeses.

The presence of a wide range of tetracycline and erythromycin resistance genes in microbial populations from different environments has been reported elsewhere [13-15, $37,38]$. In the present study, the most abundant tet and erm genes were tet $(\mathrm{S})$, tet $(\mathrm{W})$, tet $(\mathrm{M})$, and $\operatorname{erm}(\mathrm{B})$. In addition, $\operatorname{erm}(\mathrm{F})$ was widespread among the Italian cheeses.

The detection of tet and erm resistance genes by qPCR has frequently been used to monitor AR gene loads in different environments [13, 39-41]. However, it has been little used with dairy products [14, 37]. In the present work, great variation was seen between the cheeses in terms of the total abundance of $\operatorname{Tet}^{\mathrm{r}}$ and $\mathrm{Erm}^{\mathrm{r}}$ genes. Absolute copy numbers higher than $5 \log _{10}$ units have previously been reported for different tet genes [37]. In the present work, gene copy numbers were much higher than the corresponding AR bacterial counts in antibiotic-containing media. Similar results have recently been reported for tet(A) and tet(B) in hake using qPCR and Taqman probes [42]. This might be a reflection of the actual copy number of the genes in AR bacteria (e.g., they may be present in high copy number plasmids) or due to the fact that DNA-based techniques do not distinguish between live, viable-but-noncultivable, and dead bacteria $[13,14,37$, 42]. Despite this, qPCR results showed a similar trend to plate counting. Lower gene copy numbers and AR bacterial counts were always recorded for the Italian cheeses. Intragenic 
variability, amplification yields by different primer pairs, and/or the high qPCR detection limit (of $10^{4}$ copies per gram of cheese) might explain the discrepancies between the results obtained by this technique and those by gene-specific PCR.

As mentioned earlier, in addition to its use for tracking bacterial populations, the DGGE technique has already been used to analyse the polymorphism of tetracycline resistance genes in several environments $[15,16]$. However, to our knowledge, this is the first attempt to use this molecular tool to estimate the diversity of tet genes in dairy products. A canonical tet $(\mathrm{W})$ and tet $(\mathrm{M})$ sequence accounted for the majority of genes in all cheeses. Nevertheless, singlenucleotide differences at two positions in the analysed segment of tet $(\mathrm{W})$ were noted. Whether polymorphic sequences are carried by different bacterial species remains unknown. Nevertheless, the low sequence divergence suggests that tet resistance genes in cheese have a monophyletic origin and are spread among the majority bacterial populations through horizontal transfer. This has already been reported for tet genes in bacterial populations from other environments [43, 44].

\section{Conclusions}

The Spanish and Italian cheeses analyzed in this work showed wide variation in their AR bacterial populations, AR gene diversity, and AR gene loads for resistance to tetracycline and erythromycin. The results of this research are of basic and applied interest. Methods and techniques can be extended to study resistance against other groups of antibiotics of currently higher clinical significance, such as $\beta$-lactams and aminoglycosides. On average, more than three different AR genes were detected in every cheese type. Indeed, all eight erm and tet resistance genes searched for were identified in one Spanish cheese (De La Mesta) and seven in one Italian cheese (Asiago). The diversity of genes and their large copy numbers can be considered as a biological hazard with a likely, yet undefined, risk of horizontal transfer. To fully assess this risk would require the genetic characteristics of the bacteria carrying these AR genes and the location of these genes in the genome (chromosome, plasmid, transposon, integrons, etc.) to be known. As some other foods of animal origin, cheeses might be key players on the spread of AR genes via the food chain. This is certainly a biological hazard, which anticipates a transfer to susceptible bacteria during cheese manufacture or after consumption. Therefore, improvements in hygiene in animal husbandry, milk production, and cheese manufacturing practices may contribute to preventing the spread of these (and maybe others) AR determinants.

\section{Conflict of Interests}

The authors declare that there is no conflict of interests regarding the publication of this paper.

\section{Acknowledgments}

The study was partially supported by a Spain-Italy bilateral collaboration program (Ref. IT2009-0080 and IT105MD12L).
Financial support was further provided by projects from CICYT (Ref. AGL2011-24300-ALI) and INIA (Ref. RM201100005-00-00). A. B. Flórez and S. Delgado were supported by research contracts under Juan de la Cierva Program (Ref. JCI-2010-07457 and JCI-2008-02391, resp.). A. Alegría was awarded a scholarship of the Severo Ochoa program from FICYT (Ref. BP08-053). A. Quesada, Departamento de Biotecnología, Universidad de Extremadura, Spain, is greatly acknowledged for providing the authors with the $B$. fragilis 79a strain carrying $\operatorname{erm}(\mathrm{F})$.

\section{References}

[1] J. Davies and D. Davies, "Origins and evolution of antibiotic resistance," Microbiology and Molecular Biology Reviews, vol. 74, no. 3, pp. 417-433, 2010.

[2] V. M. D’Costa, C. E. King, L. Kalan et al., "Antibiotic resistance is ancient," Nature, vol. 477, no. 7365, pp. 457-461, 2011.

[3] G. D. Wright, "The antibiotic resistome: the nexus of chemical and genetic diversity," Nature Reviews Microbiology, vol. 5, no. 3, pp. 175-186, 2007.

[4] S. B. Levy and B. Marshall, "Antibacterial resistance worldwide: causes, challenges and responses," Nature Medicine, vol. 10, supplement 12, pp. S122-S129, 2004.

[5] A. H. A. M. van Hoek, D. Mevius, B. Guerra, P. Mullany, A. P. Roberts, and H. J. M. Aarts, "Acquired antibiotic resistance genes: an overview," Frontiers in Microbiology, vol. 2, article 203, 2011.

[6] D. J. Donoghue, "Antibiotic residues in poultry tissues and eggs: human health concerns?" Poultry Science, vol. 82, no. 4, pp. 618621, 2003.

[7] G. M. Durán and D. L. Marshall, "Ready-to-eat shrimp as an international vehicle of antibiotic-resistant bacteria," Journal of Food Protection, vol. 68, no. 11, pp. 2395-2401, 2005.

[8] H. H. Wang, M. Manuzon, M. Lehman et al., "Food commensal microbes as a potentially important avenue in transmitting antibiotic resistance genes," FEMS Microbiology Letters, vol. 254, no. 2, pp. 226-231, 2006.

[9] X. Li and H. H. Wang, "Tetracycline resistance associated with commensal bacteria from representative ready-to-consume deli and restaurant foods," Journal of Food Protection, vol. 73, no. 10, pp. 1841-1848, 2010.

[10] V. Perreten, F. Schwarz, L. Cresta, M. Boeglin, G. Dasen, and M. Teuber, "Antibiotic resistance spread in food," Nature, vol. 389, no. 6653, pp. 801-802, 1997.

[11] K. P. Scott, C. M. Melville, T. M. Barbosa, and H. J. Flint, "Occurrence of the new tetracycline resistance gene tet (W) in bacteria from the human gut," Antimicrobial Agents and Chemotherapy, vol. 44, no. 3, pp. 775-777, 2000.

[12] M. S. Ammor, A. B. Flórez, A. H. A. M. van Hoek et al., "Molecular characterization of intrinsic and acquired antibiotic resistance in lactic acid bacteria and bifidobacteria," Journal of Molecular Microbiology and Biotechnology, vol. 14, pp. 6-15, 2008.

[13] M. S. Smith, R. K. Yang, C. W. Knapp et al., "Quantification of tetracycline resistance genes in feedlot lagoons by real-time PCR," Applied and Environmental Microbiology, vol. 70, no. 12, pp. 7372-7377, 2004.

[14] M. Y. Manuzon, S. E. Hanna, H. Luo, Z. Yu, W. J. Harper, and H. H. Wang, "Quantitative assessment of the tetracycline resistance 
gene pool in cheese samples by real-time TaqMan PCR,' Applied and Environmental Microbiology, vol. 73, no. 5, pp. 1676-1677, 2007.

[15] R. I. Aminov, N. Garrigues-Jeanjean, and R. I. Mackie, "Molecular ecology of tetracycline resistance: development and validation of primers for detection of tetracycline resistance genes encoding ribosomal protection proteins," Applied and Environmental Microbiology, vol. 67, no. 1, pp. 22-32, 2001.

[16] J. C. Chee-Sanford, R. I. Aminov, I. J. Krapac, N. GarriguesJeanjean, and R. I. Mackie, "Occurrence and diversity of tetracycline resistance genes in lagoons and groundwater underlying two swine production facilities," Applied and Environmental Microbiology, vol. 67, no. 4, pp. 1494-1502, 2001.

[17] Z. Yu, F. C. Michel Jr., G. Hansen, T. Wittum, and M. Morrison, "Development and application of real-time PCR assays for quantification of genes encoding tetracycline resistance," Applied and Environmental Microbiology, vol. 71, no. 11, pp. 6926-6933, 2005.

[18] M. L. Diaz-Torres, R. McNab, D. A. Spratt et al., "Novel tetracycline resistance determinant from the oral metagenome," Antimicrobial Agents and Chemotherapy, vol. 47, no. 4, pp. 14301432, 2003.

[19] L. A. Seville, A. J. Patterson, K. P. Scott et al., "Distribution of tetracycline and erythromycin resistance genes among human oral and fecal metagenomic DNA," Microbial Drug Resistance, vol. 15, no. 3, pp. 159-166, 2009.

[20] M. O. A. Sommer, G. Dantas, and G. M. Church, "Functional characterization of the antibiotic resistance reservoir in the human microflora," Science, vol. 325, no. 5944, pp. 1128-1131, 2009.

[21] A. J. Patterson, R. Colangeli, P. Spigaglia, and K. P. Scott, "Distribution of specific tetracycline and erythromycin resistance genes in environmental samples assessed by macroarray detection," Environmental Microbiology, vol. 9, no. 3, pp. 703-715, 2007.

[22] S. Delgado, F. Fracchetti, B. Mayo, and S. Torriani, "Development and validation of a multiplex PCR-based DNA microarray hybridisation method for detecting bacterial antibiotic resistance genes in cheese," International Dairy Journal, vol. 21, no. 3, pp. 149-157, 2011.

[23] K. J. Forsberg, A. Reyes, B. Wang, E. M. Selleck, M. O. A. Sommer, and G. Dantas, "The shared antibiotic resistome of soil bacteria and human pathogens," Science, vol. 337, no. 6098, pp. 1107-1111, 2012.

[24] P. Mullany, E. Allan, and P. J. Warburton, “Tetracycline resistance genes and mobile genetic elements from the oral metagenome," Clinical Microbiology and Infection, vol. 18, no. 4, pp. 58-61, 2012.

[25] T. M. Barbosa, K. P. Scott, and H. J. Flint, "Evidence for recent intergeneric transfer of a new tetracycline resistance gene, tet $(\mathrm{W})$, isolated from Butyrivibrio fibrisolvens, and the occurrence of tet $(\mathrm{O})$ in ruminai bacteria," Environmental Microbiology, vol. 1, no. 1, pp. 53-64, 1999.

[26] D. Gevers, G. Huys, and J. Swings, "In vitro conjugal transfer of tetracycline resistance from Lactobacillus isolates to other gram-positive bacteria," FEMS Microbiology Letters, vol. 225, no. 1, pp. 125-130, 2003.

[27] M. C. Roberts, W. O. Chung, D. Roe et al., "Erythromycinresistant Neisseria gonorrhoeae and oral commensal Neisseria spp. carry known rRNA methylase genes," Antimicrobial Agents and Chemotherapy, vol. 43, no. 6, pp. 1367-1372, 1999.
[28] S. E. Denman and C. S. McSweeney, "Development of a realtime PCR assay for monitoring anaerobic fungal and cellulolytic bacterial populations within the rumen," FEMS Microbiology Ecology, vol. 58, no. 3, pp. 572-582, 2006.

[29] A. B. Flórez, S. Delgado, and B. Mayo, "Antimicrobial susceptibility of lactic acid bacteria isolated from a cheese environment," Canadian Journal of Microbiology, vol. 51, no. 1, pp. 51-58, 2005.

[30] A. B. Flórez and B. Mayo, "Microbial diversity and succession during the manufacture and ripening of traditional, Spanish, blue-veined Cabrales cheese, as determined by PCR-DGGE," International Journal of Food Microbiology, vol. 110, no. 2, pp. 165-171, 2006.

[31] A. B. Flórez, M. S. Ammor, P. Álvarez-Martín, A. Margolles, and B. Mayo, "Molecular analysis of tet $(\mathrm{W})$ gene-mediated tetracycline resistance in dominant intestinal Bifidobacterium species from healthy humans," Applied and Environmental Microbiology, vol. 72, no. 11, pp. 7377-7379, 2006.

[32] A. A. Salyers, A. Gupta, and Y. Wang, "Human intestinal bacteria as reservoirs for antibiotic resistance genes," Trends in Microbiology, vol. 12, no. 9, pp. 412-416, 2004.

[33] C. Devirgiliis, A. Caravelli, D. Coppola, S. Barile, and G. Perozzi, "Antibiotic resistance and microbial composition along the manufacturing process of Mozzarella di Bufala Campana," International Journal of Food Microbiology, vol. 128, no. 2, pp. 378-384, 2008.

[34] C. L. Cain, "Antimicrobial resistance in staphylococci in small animals," Veterinary Clinics of North America: Small Animal Practice, vol. 43, no. 1, pp. 19-40, 2013.

[35] C. Zhao, H. Sun, H. Wang et al., "Antimicrobial resistance trends among 5608 clinical Gram-positive isolates in China: results from the Gram-Positive Cocci Resistance Surveillance program (2005-2010)," Diagnostic Microbiology and Infectious Disease, vol. 73, no. 2, pp. 174-181, 2012.

[36] L. L. McGowan-Spicer, P. J. Fedorka-Cray, J. G. Frye, R. J. Meinersmann, J. B. Barrett, and C. R. Jackson, "Antimicrobial resistance and virulence of Enterococcus faecalis isolated from retail food," Journal of Food Protection, vol. 71, no. 4, pp. 760-769, 2008.

[37] X. Li, Y. Li, V. Alvarez, W. J. Harper, and H. H. Wang, "Effective antibiotic resistance mitigation during cheese fermentation," Applied and Environmental Microbiology, vol. 77, no. 20, pp. 7171-7175, 2011.

[38] H. H. Wang and D. W. Schaffner, "Antibiotic resistance: how much do we know and where do we go from here?" Applied and Environmental Microbiology, vol. 77, no. 20, pp. 7093-7095, 2011.

[39] J. Chen, Z. Yu, F. C. Michel Jr., T. Wittum, and M. Morrison, "Development and application of real-time PCR assays for quantification of erm genes conferring resistance to macrolideslincosamides-streptogramin B in livestock manure and manure management systems," Applied and Environmental Microbiology, vol. 73, no. 14, pp. 4407-4416, 2007.

[40] S.-M. Kim, H. C. Kim, and S.-W. S. Lee, "Characterization of antibiotic resistance determinants in oral biofilms," Journal of Microbiology, vol. 49, no. 4, pp. 595-602, 2011.

[41] L. Zhang, D. Kinkelaar, Y. Huang, Y. Li, X. Li, and H. H. Wang, "Acquired antibiotic resistance: are we born with it?" Applied and Environmental Microbiology, vol. 77, no. 20, pp. 7134-7141, 2011.

[42] M. Guarddon, J. M. Miranda, J. A. Rodríguez, B. I. Vázquez, A. Cepeda, and C. M. Franco, "Real-time polymerase chain reaction for the quantitative detection of tet $\mathrm{A}$ and tetB bacterial 
tetracycline resistance genes in food," International Journal of Food Microbiology, vol. 146, no. 3, pp. 284-289, 2011.

[43] L. Feld, S. Schjørring, K. Hammer et al., "Selective pressure affects transfer and establishment of a Lactobacillus plantarum resistance plasmid in the gastrointestinal environment," Journal of Antimicrobial Chemotherapy, vol. 61, no. 4, pp. 845-852, 2008.

[44] L. Jacobsen, A. Wilcks, K. Hammer, G. Huys, D. Gevers, and S. R. Andersen, "Horizontal transfer of tet(M) and erm(B) resistance plasmids from food strains of Lactobacillus plantarum to Enterococcus faecalis $\mathrm{JH} 2-2$ in the gastrointestinal tract of gnotobiotic rats," FEMS Microbiology and Ecology, vol. 59, no. 1, pp. 158-166, 2007. 

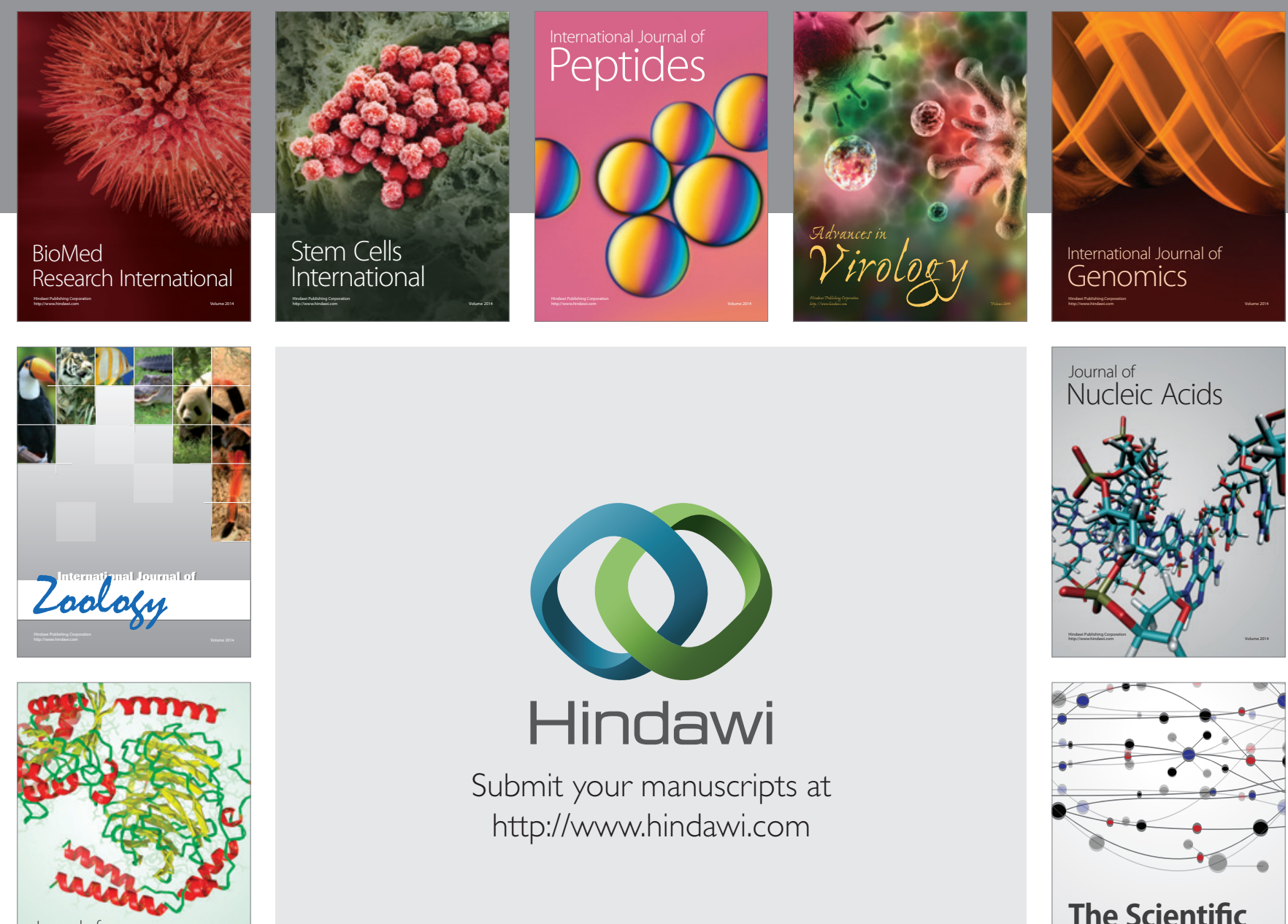

Submit your manuscripts at

http://www.hindawi.com

Journal of
Signal Transduction
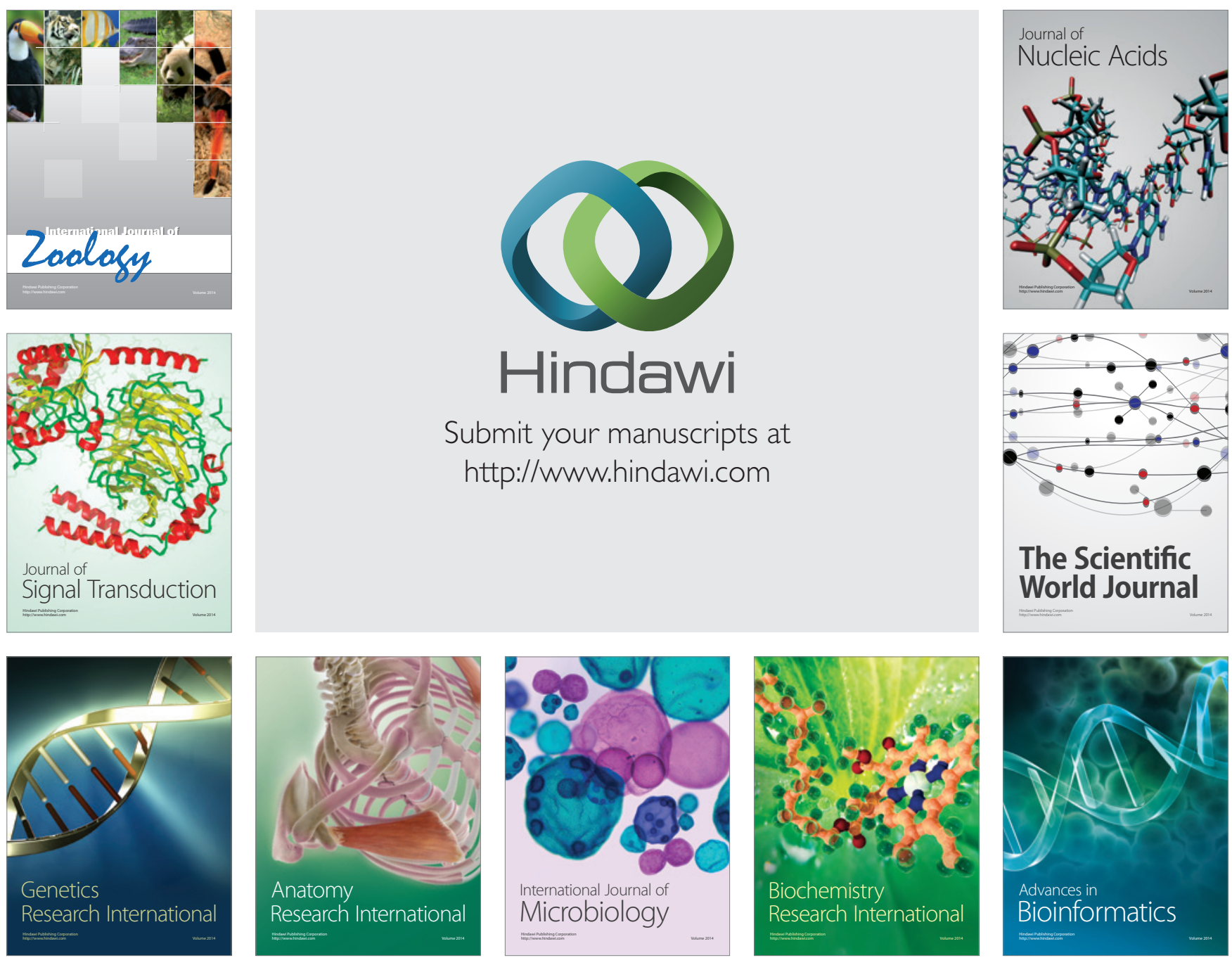

The Scientific World Journal
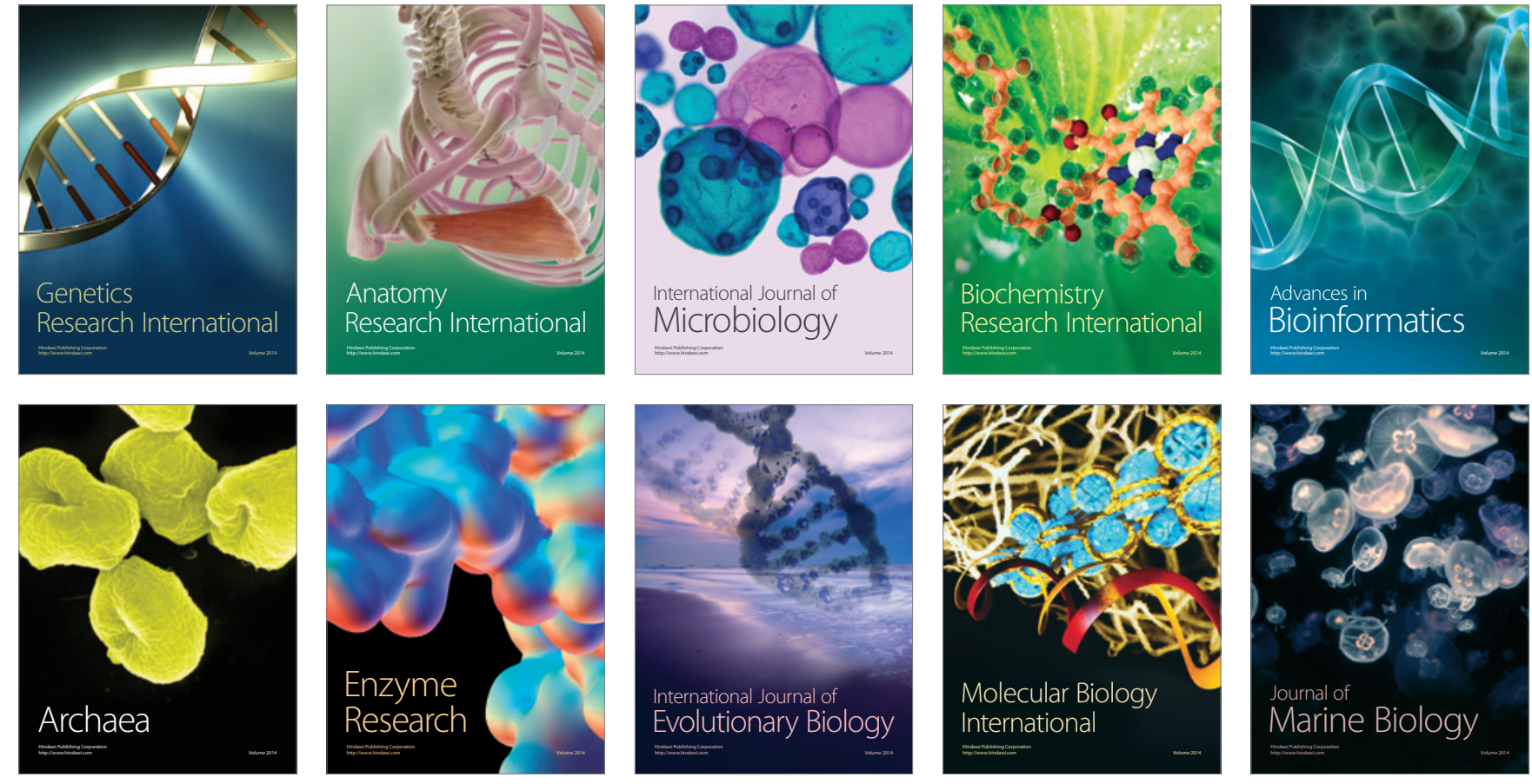